\title{
TV/Series
}

$5 \mid 2014$

Religions en série

\section{Esprit de corps, matière d'âme : les cadavres exquis du petit écran (The Walking Dead, Bones, CSI, Dexter, The Closer, et quelques autres)}

\section{Emmanuelle Delanoë-Brun}

\section{(2) OpenEdition \\ Journals \\ Édition électronique \\ URL : http://journals.openedition.org/tvseries/442 \\ DOI : $10.4000 /$ tvseries.442 \\ ISSN : 2266-0909 \\ Éditeur \\ GRIC - Groupe de recherche Identités et Cultures}

Référence électronique

Emmanuelle Delanoë-Brun, "Esprit de corps, matière d'âme : les cadavres exquis du petit écran (The Walking Dead, Bones, CSI, Dexter, The Closer, et quelques autres)», TV/Series [En ligne], 5 | 2014, mis en ligne le 01 mai 2014, consulté le 20 avril 2019. URL : http://journals.openedition.org/tvseries/442 ; DOI : $10.4000 /$ tvseries. 442

\section{(c) $($ i) $(9)$}

$T V /$ Series est mis à disposition selon les termes de la licence Creative Commons Attribution - Pas d'Utilisation Commerciale - Pas de Modification 4.0 International. 


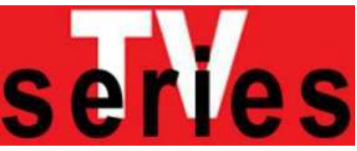

\title{
Esprit de corps, matière d'âme : les cadavres exquis du petit écran (The Walking Dead, Bones, CSI, Dexter, The Closer, et quelques autres)
}

\author{
Emmanuelle DELANOË-BRUN
}

\begin{abstract}
À l'heure où la mort, interdite, est entrée en crise dans les sociétés occidentales, on peut s'interroger sur la multiplication des cadavres dans les séries télévisées. Plus le corps se sublime et s'abstrait des outrages du temps dans ses représentations idéalisées, plus le cadavre le rappelle à son avenir répugnant. Un même questionnement de la matière humaine et de la transcendance incertaine du corps se fait ici jour, une même investigation obsessionnelle de la matière corporelle à la recherche de l'âme, de l'esprit, de cette dimension d'aspiration à un au-delà du physique et du matériel qui pourrait faire la spécificité ultime de l'homme, alors même que technique, technologie et sciences tendent à le ramener à un ensemble de procédures neurobiologiques et d'automatismes comportementaux. C'est cette tension qu'explore cet article, ouvrant des pistes interprétatives à cette résurgence du memento mori en version télévisuelle
\end{abstract}

$\mathrm{D}$ epuis une trentaine d'années, le corps envahit la série télévisée. Les séries médicales en font la vedette, qui auscultent ses multiples dérangements, tout en faisant de l'hôpital un microcosme du grand corps social et un nouveau laboratoire des passions humaines, une tranche d'humanité sociale à glisser sous le microscope de la fiction télévisuelle. Leur succès s'explique sans mal : « rien ne touche plus le spectateur qu'une fiction fondée sur l'unique réalité commune à tous les humains - celle du corps », rappelle Martin Winkler ${ }^{1}$, ce corps que le vingtième siècle a progressivement constitué en sanctuaire fragile de notre individualité ${ }^{2}$, mais dont l'hôpital rappelle l'inscription dans une économie tout aussi fragile de la santé. Plus curieuse en revanche est la fascination récente exercée par son corollaire mort, le cadavre, sur l'imaginaire télévisuel récent, tel qu'elle s'exprime en particulier dans nombre de séries américaines dont il sera question ici33. Objet de piété et de crainte mêlés, le cadavre investit progressivement les séries télévisées dans les années 90. On l'honore, on le pleure, mais on le craint aussi. Déguisé, embaumé, embelli, dans 6 Feet Under (HBO, 2001-2005), le cadavre n'est exhibé que pour mieux cacher son travail de dégradation, de peur qu'il ne fasse retour, comme dans la toute récente série The Walking Dead (AMC, 2010-), et

${ }^{1}$ Martin Winckler, Les Miroirs de la vie, Paris, éditions du Passage, 2002, p. 154.

${ }^{2}$ Jean-Jacques Courtine dir., Histoire du corps, t. 3, Les mutations du regard. Le XXe siècle, Paris, Seuil, 2006.

3 Il ne s'agit cependant pas d'une spécificité américaine, comme peuvent en témoigner des séries britanniques telles que Silent Witness (BBC1, 1996-), Waking the Dead (BBC, 20052011), Ripper Street (BBC1, 2012-). 
nous rappelle à notre condition de futur mort. Car le diagnostic tombe dès la saison 2 : tous, nous sommes infectés de cette mort qui nous habite, tous, de futurs cadavres en marche au milieu du cauchemar de ces cadavres en marche, symptômes d'une société qui aurait tout entière perdu son âme.

Interdite, disait Philippe Ariès4, tabou, dans notre société obsédée par le progrès, terrifiée par la régression du vieillissement, moins préoccupée de salut que de jouissance ici-bas, la mort ne s'est jamais tant vue à l'écran, dans la sphère domestique, à heures de grande écoute. Et ce n'est pas tant là son administration qui préoccupe, autrement dit son exécution - ce ne sont après tout que des cadavres que les personnages massacrent par centaines dans The Walking Dead. Ce sont plutôt ses effets, son lent travail de corruption des chairs, dont il est question, montrant à voir une mort intime, terriblement " transférable » à nos propres projections, cadavre dont les science procedurals se délectent, avec CSI (CBS, 2000-) ou Bones (Fox, 2005). $\mathrm{Au}$ point qu'il n'existe plus une série policière dont la scène de crime et la salle d'autopsie ne figurent les décors incontournables d'une nouvelle liturgie médico-légale qui attire semaine après semaine des millions de téléspectateurs.

Une telle fascination ne manque pas d'étonner, tant la mort se fait aujourd'hui toujours plus invisible, plus inconvenante et obscène, jusque dans la réticence et les controverses dont elle fait l'objet dans le traitement médiatique des conflits. Mais plus la mort s'abstrait, plus elle peine aussi à se faire penser, dans ce qu'Edgar Morin a appelé la grande « crise de la mort 5 » qu'a ouvert la modernité individualiste, plus elle s'affiche sur les écrans de notre quotidien. Une mort démultipliée, grossie, grouillante, proprement acharnée à détruire ce nouveau lieu d'investissement du sacré individualiste, le corps, ce corps dont Jean Baudrillard fait notre «plus bel objet de consommation », " nouveau lieu de salut », où s'investit le culte de soimême ${ }^{6}$. Mais un corps dont Georges Vigarello rappelle le travail de contrôle acharné dont il se doit d'être la cible, dans l'exigence contemporaine d'accéder à la beauté qui relève à présent de l'épreuve? Cette surenchère du cadavre à l'écran participe ainsi d'une double transgression: violation d'un interdit - voir la mort, le mort, le cadavre, voir jusque dans le cadavre, suivre le travail de corruption à l'œuvre - et profanation d'un sacré - le corps par ailleurs magnifié du personnage de série, corps iconique et triomphant - double transgression que j'aimerais examiner ici, pour tenter d'esquisser quelques pistes exploratoires. Il s'agira de considérer quelle forme

${ }^{4}$ Philippe Ariès, Essais sur la mort en Occident, Paris, Seuil, 1975

5 Edgar Morin, L'Homme et la mort, Paris, Seuil, 1951.

${ }^{6}$ Jean Baudrillard, La Société de consommation, Paris, Seuil, 1996.

${ }^{7}$ Georges Vigarello, Histoire de la beauté. Le corps et l'art d'embellir de la renaissance à

nos jours, Paris, Seuil, 2004. 
d'aspiration spirituelle s'exprime dans cette étonnante survivance réactualisée et popularisée de ce qui s'apparente à un memento mori, résurgence télévisuelle du transi de la renaissance ou des portraits funéraires du XIXe siècle ${ }^{8}$, un rappel à notre mortalité, dont il s'agit alors d'interroger la visée: moraliste, romantique, salutaire, ou plus prosaïquement hédoniste.

Première piste, la plus évidente : la piste cathartique, qui invite à voir dans la surenchère au cadavre corrompu le moyen d'expulser notre terreur de la mort, notre rage vengeresse à son égard. Mais une rage qui se mêle de fascination devant son interdit, sa disparition pudique des champs du quotidien et de sa représentation, sa relégation vers l'hôpital et dans le hors champ des images d'actualité9. À nouvelle obscénité, donc, nouvelle pornographie: les images d'une mort grotesque, boursouflée, excessive (une mort XXL, à l'instar des corps surdéterminés sexuellement de la pornographie) nourrissent un nouveau voyeurisme à qui elle fournit épisode après épisode un luxe de détails et de variations. Dans les années 90, le grotesque est celui du corps martien, autopsié en catimini dans un wagon top secret dans la série $X$-Files ( Nisei », 3.9, The $X$-files, Fox, novembre 1995). Mais bientôt Dana Scully (Gillian Anderson) attaque ses premiers cadavres humains, ouvrant la voie au grand show de l'autopsie dans les séries criminelles (voir figure 1)

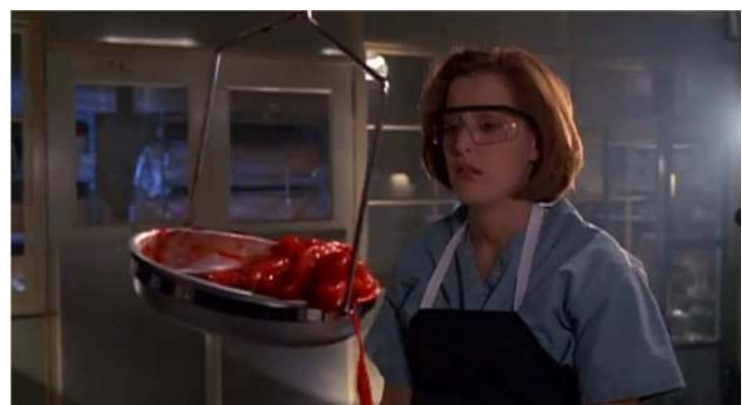

Fig. 1 : The X-Files, 5.12, « Bad Blood », février 1998

\footnotetext{
${ }^{8}$ La pratique de la photographie de cadavres, présentés dans des postures de repos ou mis en scène dans des portraits de familles, se développe au XIXe siècle aux États-Unis comme en Europe, avec l'essor des studios photographiques. Le portrait commémoratif rappelait aux vivants le souvenir des proches disparus, en particulier dans le cas de jeunes enfants. Il témoigne aussi d'une familiarité sentimentale avec la mort caractéristique de l'âge romantique.

${ }^{9}$ Les clichés d'un Weegee (1899-1968, Arthur Fellig de son vrai nom, photographe à New York dans les années 30 et 40 et spécialiste des urgences criminelles), dans la presse quotidienne, sont aujourd'hui peu imaginables, symptôme d'une asepsie qui a progressivement gagné l'univers médiatique dans son traitement de la violence urbaine.
} 
La scène, surprenante sur petit écran au terme des années 90, désacralise un corps dont les organes sanguinolents atterrissent un à un dans une balance pour la pesée, s'échappent, grassement indignes, sous l'œil blasé de la légiste. Dès 2000 CSI, avec rien moins que deux intrigues par épisode - donc deux victimes au moins - ouvre le catalogue apparemment sans fin des outrages faits ou subis par les corps dont les divers états de fraîcheur et les multiples blessures sont observés à la loupe. Avide de tout voir et de tout montrer, la caméra fond sur les tissus pour y faire surgir la vérité des coups portés, pénètre à même les chairs avec une précipitation vorace : le CSI shot qui fait le succès des premières saisons, nous plonge au plus atomique de la matière et invite ouvertement le spectateur au voyeurisme, sous couvert d'éclaircissement scientifique. Mangé par les vers, gorgé d'eau, découpé en morceaux, déchiqueté par les coyotes, pulvérisé, carbonisé, réduit en purée, le cadavre affiche à peu près tous les états mais surtout les pires, scrupuleusement auscultés par l'équipe d'experts suréquipés. À la galerie visuelle s'ajoute la touche olfactive, le rappel de la puanteur cadavérique et de sa raison: la fermentation interne des gaz qui boursouflent les chairs vites corrompues, l'odeur pestilentielle de la " soupe humaine " qu'est devenu un cadavre d'avoir séjourné dans un sac en plein soleil, et qui s'accroche aux cheveux de Sara Sidle (Jorja Fox) dans « Bully for You » (CSI, 2.4, octobre 2001).

En 2005, Bones fait entrer le squelette dans la danse, et son enveloppe de chair desséchée, qu'il faut parfois enfiler, littéralement, réhydratée, pour en collecter les empreintes. Et bientôt, le police procedural prend le relais. C'est le cas par exemple du pourtant très sage The Closer (TNT, 2005-12012), dont l'épisode pilote s'ouvre sur la découverte d'un cadavre boursoufflé au crâne partiellement défoncé, dont la caméra se rapproche complaisamment, tout comme son héroïne au look de belle du Sud moderne (voir figure 2).

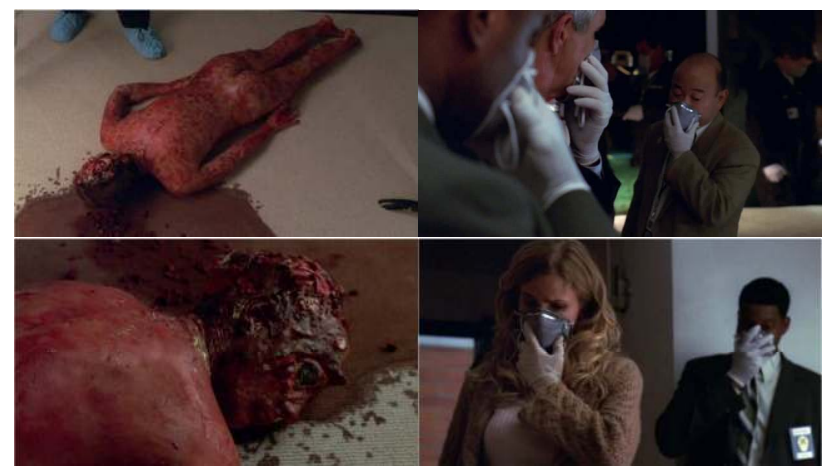

Fig. 2 : The Closer, 1.1, juin 2005 
Régulièrement, le même motif revient d'une mort abjecte mais sur laquelle la caméra s'attarde, comme fascinée de découvrir ce qui jamais n'est donné à voir, tout l'interdit d'une société hygiéniste et narcissique $^{10}$ : un corps grossièrement organique, corps de fluides malodorants, de gaz et de matières gluantes et visqueuses, sous la parfaite enveloppe de peau et les tenues ajustées dont les personnages alentour rappellent la splendeur perdue (voir figure 3).

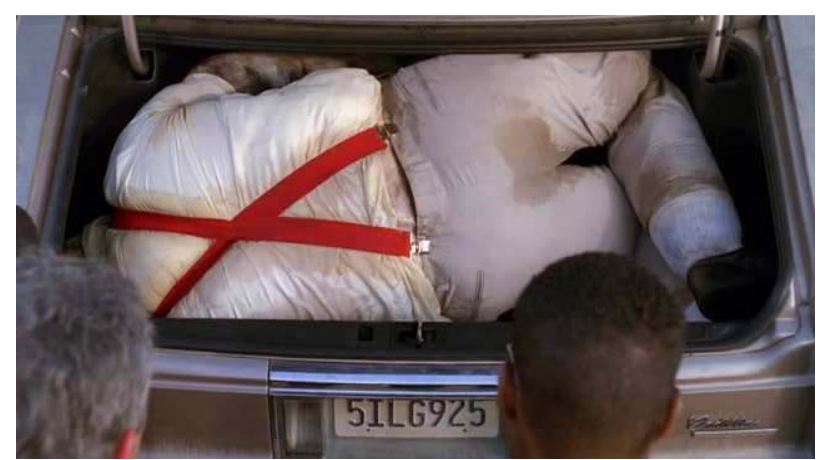

Fig. 3 : The Closer, 4.12, « Junk in the Trunk ${ }^{11}$ », février 2009

Sous le sanctuaire de l'enveloppe corporelle, dans cette nouvelle fabrique de la perfection esthétique, hygiénique et fonctionnelle qu'est le corps-objet de la société de consommation, on rêve d'aller voir, de soulever en douce le capot de peau ${ }^{12}$, comme les chirurgiens de Nip/Tuck (FX, 2003-2010), afin le cas échéant de re-souder l'âme, ou au pire de lui assurer la paix éternelle, mais en tous cas de jouir, purgés de notre effroi, de l'interdit ainsi bravé. Jouir du corps mortel, du cadavre interdit, c'est en exorciser à la fois l'attirance coupable et le dégoût, la peur, y re-médier par la médiatisation indispensable qui en fait spectacle, en orchestre la comédie, dans le bal des masques respiratoires, la chorégraphie des professionnels du crime, jusqu'à la

\footnotetext{
${ }^{10}$ Dans l'épisode suivant de la saison 1, c'est une ex-mannequin qui est retrouvée morte dans sa douche, mannequin dont les portraits avantageux ornent les murs de la maison à présent envahie d'officiers de police.

${ }^{11}$ Le titre de l'épisode ajoute le sarcasme à l'outrage cadavérique : l'expression « junk in the trunk », qui désigne des fesses bien rebondies, s'applique ici au cadavre gonflé de gaz d'un homme devenu « ordure » (junk), jeté au fond d'un coffre (trunk). L'ironie déflationniste dénie au cadavre toute superbe, que les auréoles et les boursouflures lui ôtent clairement à l'image.

${ }^{12}$ La métaphore mécanique est inspirée de la belle analyse que Thibaud de Saint Maurice effectue de la série dans Philosophie en séries, Paris, Ellipses, 2009. Nip/Tuck ravive ici le souvenir ancien des liens entre art et anatomie, entre scènes de théâtre et amphithéâtres de dissection, dans cette auscultation du corps et des âmes, qui par exemple fait chez Shakespeare dialoguer le vif et le mort dans la célèbre scène de l'ossuaire qui confronte Hamlet et le crâne de Yorick (Hamlet, acte V, scène 1).
} 
bouffonnade macabre d'une morgue surchargée où l'enquêtrice cherche en vain son cadavre numéroté (voir figure 4).

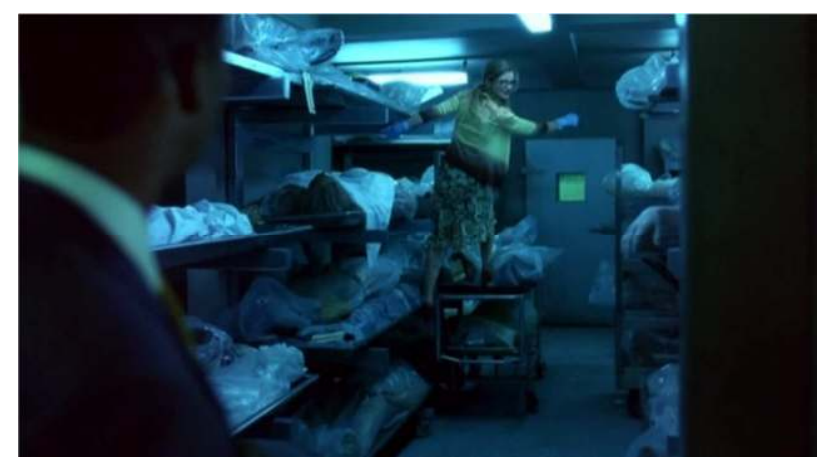

Fig. 4 : The Closer 1.3, « The Big Picture », juin 2005

Toujours présente dans The Closer, la caméra se montre qui représente l'horreur, tandis que Buzz ${ }^{13}$, le technicien (Philip P. Keene), filme les scènes du crime, se repaît des détails du corps dégradé pour mieux par contraste sublimer le vivant, l'efficacité du geste, la fulgurance de l'esprit, la hauteur splendide face à l'abjection.

The Walking Dead pousse jusqu'à l'extrême cette forme de pornographie morbide cathartique, principe même sur lequel la série est bâtie : c'est la délectation de la mort répugnante, son spectacle tout entier, démultiplié, qui nous est offert, sous la forme de foules compactes qui se déplacent à un rythme d'automates. Et face aux cadavres d'autant plus révoltants qu'ils refusent de mourir, l'érotisme des corps vivants, splendides, arrogants de perfection physique et de force, s'affirme : corps que les semaines passées en fin fond de forêts ou bien sans véritable eau courante dans une prison sordide ne dégradent en rien, coiffure savamment négligée, film de sueur tout juste bon à faire luire le muscle. L'athlétique Michonne (Danai Gurira), à partir de la saison 2, comme Daryl (Norman Reedus) dès la saison 1, est un exemple de ces corps splendides, parfaites machines de muscles huilés malgré les conditions extrêmes de leur environnement (voir figures 4 et 5 ).

${ }^{13}$ On lit évidemment dans le surnom du personnage l'image de l'insecte qui bourdonne autour du cadavre dont il se nourrit, autant que l’idée de la rumeur médiatique. 


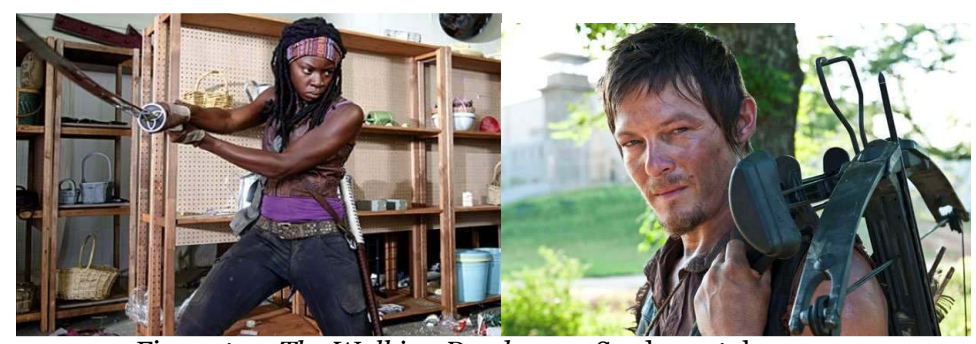

Fig. 4 et 5 : The Walking Dead, 3.1, « Seed », octobre 2012

L'outil parfait de ce corps magnifié en objet de désir occis du mort sans discontinuer, dans un déchaînement libérateur de violence: chaque épisode apporte son lot de massacres, sa surenchère d'exécutions, dans une logique de progression narrative proche du jeu vidéo ${ }^{14}$. La jouissance, elle, nait de ce spectacle conjoint de mort et de désir, spectacle d'une consommation sans fin du corps, vivant ou mort, désirable ou abject, pris dans une économie du gaspillage. D'un côté, des personnages organisés en communauté, et singularisés, offrent leur beauté et leur efficacité aux regards, ainsi qu'une vision magnifiée du corps social : le groupe de survivants obéît à un parfait équilibrage générique, ethnique et générationnel, et compte, toutes saisons confondues, son patriarche blanc lettré, son shérif texan, son Coréen débrouillard, sa blonde pétroleuse au grand cœur et sa jumelle éthérée maternante, son Sudiste red-neck, sa noire athlétique - sujet token ${ }^{15}$, exemplaire d'une identité d'affichage, dans une conception communautariste de l'être. De l'autre côté, des masses de zombies indéterminés déclinent la panoplie des corps déchiquetés et putrescents. Dans cette confrontation, la catharsis prend une dimension supplémentaire, en réaffirmant dans le combat du groupe singulier contre la foule anonyme des morts-vivants la primauté de l'individu, la victoire - si fragile fut-elle - du sujet organisé en société aux fonctions bien balisées. À ceci près que le sujet se fait ici plutôt étendard d'un stéréotype volontairement représentatif, objet de projections identitaires idéalisées dessinant l'utopie d'une société racialement et socialement intégrée, dans la dystopie de sa décomposition

${ }^{14}$ L'épisode de l'exécution des zombies libérés d'une grange dans « Pretty Much Dead » (2. 7) concentre la formule de confrontation propre à la série, opposant le groupe de survivants, splendides, filmés en contre plongée dramatique, et disposé en peloton d'exécution, et d'anciens proches à présent morts-vivants, qui s'avancent vers le groupe pour être massacrés.

${ }^{15}$ Un personnage est dit «token » quand il semble planté pour répondre à une attente de diversité culturelle, notamment concernant les noirs américains. On parle volontiers en français du « noir de service». 
Mais c'est aussi que, précisément, dans cette avalanche de cadavres, se pose la question de l'individu, de sa singularité, de sa résistance à la foule anonyme des morts qui s'accumulent, tant dans le fantasme post-apocalyptique de The Walking Dead que dans les étagères des morgues, où les morts font clairement concurrence aux vivants. Si les séries nous repaissent de la peur de notre devenir cadavre pour mieux nous en expurger un temps, c'est aussi pour mieux tenter d'exhumer l'individu d'une économie de la consommation et de la représentation qui en a soldé l'essence. C'est alors ici une piste ontologique qui s'ouvre, où le cadavre se fait lieu d'investigation en quête d'une âme dont l'univers représenté semble avoir perdu le sens. Autour de la table de dissection sur laquelle il trône, des personnages en déficit d'âme s'agitent, tueurs comme paléontologues psychopathes (le héros éponyme dans Dexter, incarné par Michael C. Hall, Temperance Brenan dans Bones jouée par Emily Deschanel), entomologistes à tendance hermitique (Bill Grissom incarné par William Petersen dans $C S I$ ), techniciens du crime dépourvus de toute intimité propre, en dehors d'un univers professionnel magnifié qui leur tient lieu de repère identitaire. Objet de toutes les craintes et de toutes les attentions, le cadavre focalise l'inquiétude d'une perte de l'individualité dont l'âme fut longtemps la métaphore magnifiée, celle dont la perte, plus que la corruption des corps, inquiète.

Réfugiés au fond d'un bunker, à la fin de la $1^{\text {ère }}$ saison de The Walking Dead, la poignée de rescapés du charnier ambiant, les yeux rivés sur les images d'un IRM, écoute un scientifique commenter les images d'un cerveau en activité, fasciné par le feu d'artifice des synapses en pleine effervescence. La dramaturgie visuelle donne toute sa solennité au passage: le décor kubricien, mélange de $D r$ Strangelove et de 2001, A Space Odyssey ${ }^{16}$, le lent mouvement de resserrement du cadre, depuis le plan large jusqu'aux gros plans sur les visages bouleversés, les contreplongées sur des silhouettes graves, tandis qu'à l'écran, dans un effet de mise en abyme, se joue le drame d'une mort sous IRM (voir figure 6).

${ }^{16}$ Le dialogue qui se noue entre le docteur Jenner (Noah Emmerich) et l'ordinateur relié au vidéoprojecteur redouble cette résonance. 


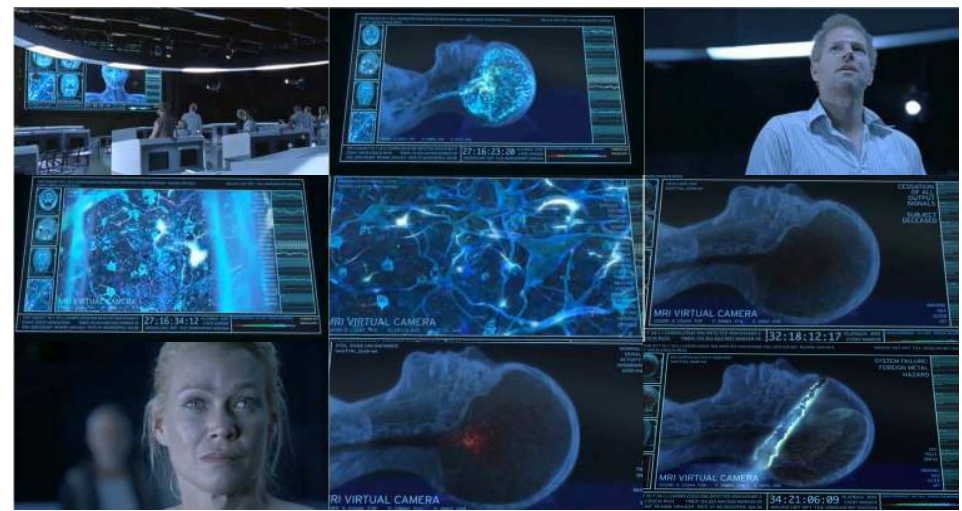

Fig. 6 : The Walking Dead, 1.6, « TS-19 », décembre 2010

Le commentaire du docteur Jenner (Noah Emmerich) accompagne cette radiographie d'une mort annoncée et d'une résurrection monstrueuse, débrouillant l'écheveau qui lie le vivant au corporel, l'âme lumineuse à la matière opaque :

Somewhere, in all that organic wiring, all these ripples of light, is you, the things that make you unique and human. Those are synapses, electric impulses in the brain that carry all the messages. They determine everything a person says, does, or thinks from the moment of birth, to the moment of death. (The Walking Dead, 1, TS-19, décembre 2010)

L'IRM se fait caméra, zoome sur les étincelles qui représentent la vivacité d'une activité cérébrale, avant que la mort n'y déploie son voile obscur. Sous la modernité technologique de la représentation, le registre de la lumière, de l'incandescence et de l'obscurité ravive la mystique immémoriale qui tire l'âme mystérieuse vers la lumière et la quête de la vérité, l'espoir du bien. Mais bientôt le cadavre s'anime, envahi par le mal curieux de cette mort qui se survit, jusqu'à ce qu'une balle salvatrice traverse le crâne de part en part.

Avec le groupe de réfugiés abasourdi, nous avons assisté à la mort en direct d'un être devenu mort vivant. Nous y avons assisté au plus près, dans la vérité scientifique de la matière, grâce à une imagerie magnétique capable de traverser les chairs, de percer nos enveloppes. Nous avons vu s'éteindre une lumière, nous avons vu l'obscurité s'emparer du vif, puis la mort vivante se faire re-tuer, nous avons assisté à la pénétration de la balle, double viol métaphorique de l'âme et de l'image. De tous les cadavres terrifiants de The Walking Dead, c'est peut être celui-là, sans corps, sans identité d'enveloppe physique et de visage, qui reste en tête, parce qu'il déplace la mort dans le champ 
de l'immatériel, de l'âme, vaguement accessible à la science, mais toujours mystérieuse ${ }^{17}$. Sans doute aussi, de toutes les images de cadavres vivants, la plus troublante, car de la mort elle a exprimé moins le travail matériel de corruption du vif que celui d'extinction immatérielle de l'être, irreprésentable sauf dans cette nouvelle forme d'abstraction du matériel qu'est l'image scientifique, cette pénétration de l'être qui l'abstrait en fonction organique, connexions nerveuses, circulation de fluides.

Que reste-t-il de l'âme dans le corps mort, s'interrogent les personnages de The Walking Dead - du moins, une saison et demie durant - qui pourrait justifier qu'on en conserve l'odieux cadavre vivant? Et quid de l'âme vive de celui qui massacre allégrement un autre qui avait été jusque-là son prochain, fille, femme, frère, ami, rattrapé par la mort ? Quid de l'individu dans son rapport à une masse informe qu'il aura tôt fait de rejoindre, quid de sa singularité, de ces lumières intérieures qui l'animent, puisque son être cadavre, son absorption dans les rangs abrutis d'une biologie corruptible, constituent son destin affiché dès la saison 2 ? La question, si elle ne hante pas bien longtemps les personnages d'une série vite rattrapée par la logique massacreuse et jouissive du jeu vidéo, rejoint le dialogue constant que les divers techniciens du crime entretiennent avec les cadavres dans la série policière.

Car c'est bien là le travail auquel s'attache avec acharnement le médecin ou le scientifique dans la série policière : retrouver dans le cadavre la singularité d'un être. Identifier le corps, retrouver sous les chairs viciées ou en passe de l'être un individu singulier, représente la première étape de tout travail d'investigation scientifico-policière représenté à l'écran. Au cadavre anonyme, il faut trouver une identité, aux organes et os maltraités par la violence ou le travail des matières, il faut raccrocher une singularité propre d'avant l'anonymat biologique. La tâche en est paradoxalement confiée à des personnages qui figurent, à des degrés divers, la psychose d'une existence dénuée d'essence, l'abstraction de l'humain dans le double topos contemporain d'une économie effrénée de la consommation et de la connaissance, soumises au même régime de l'efficacité et de la productivité. Consommation de cadavres, d'abord, que l'économie productive de la jouissance sans entrave multiplie, épisode après épisode, dans un Las Vegas qui en est devenu l'emblème, dans CSI. En regard, l'équipe d'enquêteurs scientifiques de la série, sous la houlette de l'entomologiste Bill Grissom, se compose de personnages sans vie propre à qui seule

17 L'exécution de l'enfant morte-vivante qui ouvre la saison est certes tout aussi difficile à recevoir, et sans doute aussi marquante. Mais la dimension dramatique de cette ouverture joue plus sur la surprise (l'enfant surgit dans une scène dont tout dénote la normalité apparente, tandis que le shérif Grimes s'ouvre à son adjoint de ses difficultés conjugales), que sur la solennité esthétique qui fait de la scène de l'exécution sous IRM un point d'orgue dramatique. 
importe la nécessité de produire des conclusions irréfutables. Assimilée à une entrave à l'efficacité professionnelle, l'intimité n'y a pas sa place. De Catherine Willows (Marg Helgenberger), on saura juste qu'elle a été strip-teaseuse et qu'elle élève seule une fille rescapée d'un mariage raté. Mais l'enfant, à peine entraperçue, représente tantôt un embarras domestique - il faut la faire garder - tantôt une frustration affective - la mère ne voit jamais sa fille. De Warrick (Gary Dourdan), on ne connaîtra que son addiction au jeu ; de Sara Sidle, sa solitude dépressive. Privée d'intimité, l'identité sociale du personnage demeure circonscrite à son positionnement dans l'organigramme complexe du laboratoire de la police scientifique ${ }^{18}$, et à sa capacité technique, sa connaissance ciblée d'un domaine de l'humain ou de tout ce qui le touche (entomologie, biologie, physique, balistique, mécanique, ...). Moins qu'une identité, il est une fonction herméneutique dans l'économie narrative de la série, fonction associée à un type, mais tout entière tournée vers l'efficacité scientifique. Efficacité par ailleurs surdéterminée par un décor qui la magnifie débauche d'équipement dernier cri, d'innovation technologique, affichage d'une modernité scientifique triomphante (voir figure 7). Reste que cette modernité scientifique et sociale fait l'économie de l'individu singulier au profit de l'équipe et de la répartition des tâches, qui limite la singularité individuelle à l'extrême, voire la gomme ${ }^{19}$, et multiplie la figure de ce qu'on pourrait appeler le «dedicated freak", le bourreau de travail bardé de connaissances et dévoué à sa tâche, à l'intimité reléguée aux limbes de la narration.

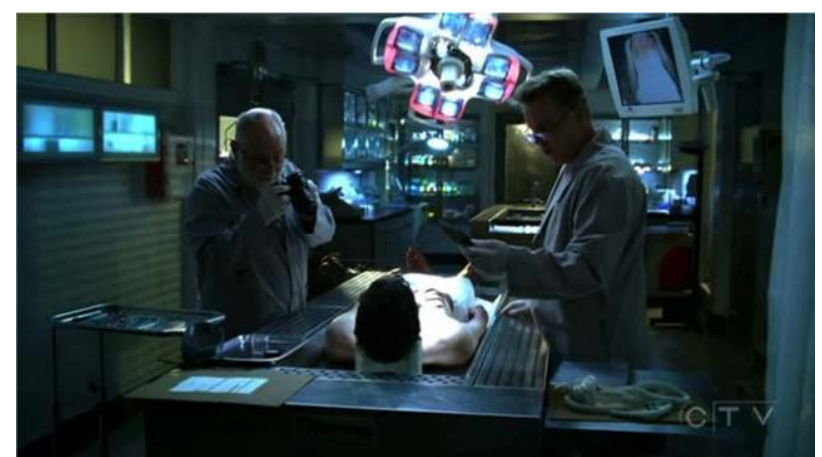

Fig. 7 : CSI, Las Vegas, 8.4, « The Case of the Cross-Dressing Carp », octobre 2007

${ }^{18}$ Les questions de promotions internes dans la série figurent en filigrane de manière récurrente dans CSI, Las Vegas ou dans Dexter, biaisant les rapports sentimentaux (amitié, amour) entre les personnages.

${ }_{19}$ Typiquement, on ne sait rien de Bill Grissom, dont le bureau relève de l'antre étrange, mi lieu domestique, mi laboratoire. 
Pour ces spécialistes des traces et indices, l'univers de l'enquête est un temple froid et sombre tout en couleurs bleutées, un purgatoire de la transparence scientifique aux parois de verre cristallines dans lequel s'évanouit l'intimité singulière de l'être, son âme, comme perdue dans l'investigation d'un monde qui semble curieusement en manquer. Aux deux extrêmes d'un spectre moral singulièrement chamboulé, le criminel (Dexter), et le savant, l'homme - la femme - de culture (Bones), on trouve le même déficit d'âme, la même expérience d'un vide émotionnel dont il n'est même pas sûr qu'ils veuillent vraiment le combler ${ }^{20}$. Entre les deux, des personnages fonction, à l'intimité réduite à la portion congrue, personnages remplaçables, consommables, dans cette excroissance fictionnelle de la société de consommation qu'est la série, série qui programme la valse des personnages sans laquelle la lassitude s'installe. Dès le pilot de CSI, une jeune recrue brune ne termine pas l'épisode, immédiatement remplacée dans l'épisode deux par son double, Sara Sidle. À chaque nouvelle saison de Dexter, ou presque, Debra (Jennifer Carpenter) rencontre l'homme de sa vie. À chaque nouvelle saison ou presque, il meurt ou disparaît de son horizon sentimental, bientôt remplacé par le suivant. À chaque nouvelle saison, le précédent est oublié, l'ardoise sentimentale effacée, le deuil cantonné à quelques répliques dont la fonction semble être de rappeler au spectateur les péripéties dramatiques précédentes. Demeurent quelques scories qui forgent le personnage en figure d'Iphigénie post-moderne prise entre un dragon et un chevalier également morts -The Ice Truck Killer (Christian Camargo), l'agent Lundy (Keith Carradine)- héroïne qui progresse en combattant force démons intérieurs à coup de jurons explosifs. Mais la série le fait en alimentant un renouvellement sentimental propre à raviver les attentes spectatoriales, jalonnant le parcours de Debra d'aventures, ou de traumatismes, capables d'assurer son efficacité dramatique. Pour le reste, Debra se console à son tapis de jogging; sexy, hot, elle figure parfaitement le sujet contemporain - sujet-objet de désir, enfermé dans un narcissisme communément partagé, dont l'individualité s'absorbe entièrement dans le travail.

Dans ces séries, le personnage de fiction représente moins une individualité psychologique qu'une fonction et un mythe : celui de sa valeur performative, de son efficacité professionnelle, incarnée dans la perfection d'un corps à la mécanique parfaitement huilée et en cela, désirable. S'il y a de la formule, dans cette construction fonctionnelle

${ }^{20}$ Criminel pour l'un, spécialiste d'anthropologie judiciaire pour l'autre, Dexter ou Temperance Brennan partagent une même paralysie affective née d'un traumatisme infantile. Suite au massacre de sa mère pour le premier ou la mort violente de ses parents pour la seconde, ils adviennent moins à la conscience qu'à son contraire, adultes purgés de toute discrimination morale et émotive. En ce sens, Dexter et Brennan figurent les Adam et Eve monstrueux et paradoxaux d’une société prédiquée sur la violence. 
du personnage - après tout, Sherlock Holmes ou Hercule Poirot avaient-il une intimité ? - leur inscription dans un univers hypertechnique soumis à une pression du résultat, et le déplacement de leur intérêt vers l'investigation du cadavre, la reconstitution d'une identité, la focalisation sur les indices qui informeront les derniers instants de sa biographie et de son drame personnel, soulignent par ricochet l'extrême évanescence du personnage à peine sujet, son obsession d'objet, ou de sujet minoré, pour le cadavre, cette ancienne matière d'être dont il traque l'existence de sujet, l'intimité, la voix, le discours, les sentiments - autrement dit, l'âme. " Talk to me ", répète, comme une mantra, la médecin légiste de CSI Miami ${ }^{21}$ (Khandi Alexander), au cadavre allongé sur sa table d'autopsie. Curieusement, c'est autour de la table d'autopsie, autour du cadavre plus ou moins répugnant, que rejaillit le discours de l'intimité, l'invitation au dialogue des âmes dans ce nouveau confessionnal improbable, le souci de percer le mystère de l'autre, d'en sonder l'être, dont l'enquêteur paraît avoir perdu le sentiment.

Car si le cadavre est devenu lieu d'investigation, l'autopsie, elle, se fait nouvel espace liturgique où s'exécute un rituel de la confession patiemment soutirée, de la ré-individuation de la victime. Un double mouvement d'abstraction du cadavre dans le discours scientifique, et de re-sacralisation de la victime, se fait jour. D'un côté, le corps où ses restes, traités en données à analyser, passés au crible des connaissances biologiques, chimiques, anthropométriques, mécaniques, soumises à l'examen des lois de la physique, de la gravité, de la résistance des matériaux, ou de la météo, modélisés pour des tests censés reproduire les conditions du crime, devient objet d'étude, matière dont la densité, la composition, la dynamique, les conditions de détérioration, sont examinés à la loupe. Et dans cette nouvelle religion de la science et du savoir dont laboratoires et bureaux flambant-neufs sont les cathédrales proposées à la dévotion des spectateurs, c'est une représentation curieuse de l'être qui émerge. Les sciences physiques sollicitées par les techniciens du crime abstraient graduellement la victime de sa structure biologique périssable, passent progressivement de l'horreur du cadavre premier à la certitude rassurante des chiffres, codes génétiques, formules chimiques ou mathématiques, graphes et autres reconstructions en $3 \mathrm{D}$ qui littéralement virtualisent le corps, dans une forme de transsubstantiation scientifique. L'évidence, l'indice (the evidence), qu'il faut suivre, reconstruit une scène, puis une existence, dont tous les paramètres sont bientôt exprimés - mais une existence paramétrée, précisément, réductible à une série d'algorithmes chimiques, aussi

${ }^{21}$ La multiplication des spin-offs marque le triomphe d'une série si bien intégrée dans cette économie de la consommation et de la reproduction qu'elle a produit une multitude de copies, aux États-Unis, en Angleterre ou en France. 
formulaïque, en d'autres termes, que la fiction dans laquelle il s'inscrit, abstraite de la matérialité biologique du cadavre. Criminal Minds, qui démarre la même année que Bones, ajoute à la palette des connaissances scientifiques déjà redoublée autour de l'exploration du squelette toute la gamme des sciences humaines (psychologie, psychopathologie, littérature, sociologie, neurosciences) et de l'informatique, mobilisées pour la traque du criminel. Les sciences cognitives, au service de la résolution du crime, complètent le portrait d'une humanité finalement bien peu singulière, réductible à des schémas d'actions et de réactions, catégorisable en types et familles un homme tout entier formulaire.

Tantôt bel objet de consommation, tantôt somme de savoirs et de méthodologies remarquables, l'humain dans la série se trouve régulièrement ramené à un ensemble de données scientifiques et psycho-pathologiques qui, dans l'apparence de sa singularité, débusquent au contraire son caractère commun, attendu, cet anonymat de la masse dont il aspire pourtant à se départir ${ }^{22}$. Les corps féminins parfaits et parfaitement interchangeables dont les plans généraux de CSI Miami régalent à maintes reprises le spectateur, modèles plastiques sanctifiés par l'environnement socialement valorisé dans lequel ils sont inscrits, en sont l'illustration caricaturale. La plastique avantageuse des enquêteurs et des scientifiques participe pourtant de la même logique qui standardise les représentations ${ }^{23}$. Dans la série de grande diffusion, véhicule d'un modèle culturel et social hautement normatif et stéréotypé, l'identité se décline sur le mode de la similitude, de la répétition, rapportée à un lot commun biologique et comportemental répertorié, rassurant de dématérialisation hygiénique. Reste que ces similitudes sont également troublantes, car affleure alors une perplexité sourde, comme l'incertitude d'une transcendance humaine singulière, perdue dans l'excès de connaissances et l'économie de la reproduction.

Mais dans le même temps, une liturgie du corps victimisé se met en place, qui opère visuellement une re-sacralisation de la victime, en corps de souffrance sublimé, corps curieusement libéré de son image désirable - un corps matière, en d'autres termes, où vient se raccrocher, dans une résurgence d'une morale très chrétienne de l'épreuve et de la souffrance, la possibilité d'une âme. Si le corps est biologique, si le cadavre en est son expression, heureusement soumis à

${ }^{22}$ Ce tiraillement entre singularité et standardisation, originalité et conformisme, régit la question de l'identité contemporaine, à la fois individualiste et massifiée. Voir à ce sujet le chapitre sur la beauté «épreuve » concue à présent comme "achèvement du sujet » et pierre d'achoppement de la singularité propre dans Histoire de la beauté, de George Vigarello, op . cit., chapitre 4, cinquième partie.

${ }^{23}$ La distribution, sémiotiquement encodée, réserve fréquemment les positions d'autorité à des figures patriarcales moins irréprochables de leur personne, pour mieux figurer leur assise intellectuelle. 
un rituel de transsubstantiation par la science, la mort, elle, est sacrée, un interdit curieusement pudique qui laisse en hors-champ son exécution, sauf dans son abstraction technique (le crâne et le cerveau sous IRM de The Walking Dead), dans des séries qui par ailleurs se délectent de ses effets ${ }^{24}$.

Le crime dans Dexter est ainsi constamment emmêlé de religiosité, de légitimation morale et rituelle. Centrée autour de la figure du criminel, la série le transforme en exercice philosophique à portée morale, sentimentale et spirituelle, par lequel se joue l'accès à une conscience sensible. Psychopathe baptisé dans le sang de sa mère massacrée à la tronçonneuse, Dexter tue des criminels que la justice ne parvient pas à punir : c'est là le code que lui a enseigné son père afin de canaliser ses pulsions criminelles. Victime devenu bourreau, orphelin monstrueux, ange meurtrier, Dexter plonge son scalpel dans la logique d'un système judiciaire axé sur la rétribution, dans l'espoir de percer le mystère des âmes sombres. Et c'est en prodiguant une mort apparemment juste que Dexter s'humanise paradoxalement, tandis que l'administration de la mort prend des allures rituelles. Ce n'est pas la moindre des ironies de cette série ouvertement provocante qui se joue d'un système dont elle démonte les rouages manifestement criminogènes, comme le rappelle le clin d'œil goguenard du héros en fin de générique 25 .

Il y a dans Dexter une liturgie du crime dont la première saison établit le cérémonial et les gestes sacralisés : tablier de boucher, masque de plexiglass, table sacrificielle sur laquelle est étalée la victime, prélèvement d'une goutte de sang glissée sur une plaque de verre, confession du criminel confronté au rappel de son crime, avant l'exécution, suggérée mais rarement montrée par un déplacement pudique de la caméra qui s'écarte de la victime. Le rituel invoque à la fois le sacrifice et la propreté : la tenue suggère la violence à venir, tandis que les bâches transparentes qui habillent un décor parfaitement blanc et fantomatique évoquent une forme de purification. Et au milieu, la victime nue, littéralement scotchée à sa table par un voile de film alimentaire judicieusement disposé, figure les anciens martyrs de l'iconographie renaissante, soumis de gré ou de force à un rituel de la confession doucereuse et violente, soutirée par une troublante figure de justicier ouvertement criminel. Le tueur de la trinité (Trinity), que traque Dexter au long de la saison 5, ou le tueur

24 The Walking Dead distingue bien la boucherie des morts vivants débinés complaisamment sous l'œil de la caméra de la mort des vivants, voilée et esthétisée, beaucoup moins visible.

25 Dexter cultive l'ambiguïté et le trouble autour de la question de la peine de mort, qu'elle semble à la fois justifier (Dexter ne tue que des criminels) et dénoncer (Dexter est un criminel, dont le contact paraît dangereusement contagieux, allant jusqu'à éveiller chez un District Attorney apparemment exemplaire les pulsions criminelles jusque-là canalisées par les règles d'administration de la justice, dans la saison 3). 
de l'apocalypse (The Doomsday Killer) auquel il est confronté dans la saison 6, redoublent le motif de cette fascination pour une mort sacrée, ritualisée, rapportée à une religion qui fait d'un cadavre en croix son emblème. Le parallélisme est directement invoqué dans la saison 6, où le Doomsday Killer finit sanglé à un autel, aux pieds d'un Christ en croix. Derrière la surenchère de provocation qui rappelle à la dimension pornographique de la fascination pour le cadavre à venir, apparait la transgression du criminel qui se fait littéralement dieu sur terre, administrateur de mort et de justice troublante dans sa violence symbolique (voir figure 8). De salut pourtant il n'est pas véritablement question : Dexter travaille d'abord la question de la justice rétributive et de son administration, ramenée à la brutalité de sa nature criminelle ; reste qu'une forme de sacralisation est à l'œuvre, au sein même de la transgression sarcastique.

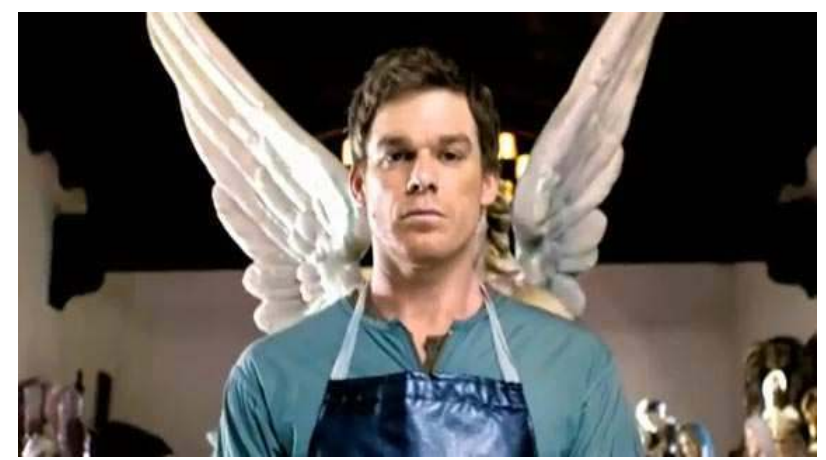

Figure 8 : Dexter, 6.12, « This is the Way the World Ends », décembre 2011

Dans CSI, comme dans The Closer, ou dans Bones, la même forme de ritualisation et de sacralisation est à l'œuvre dans l'incontournable moment de l'autopsie ou de la conversation autour des os déployés sur la table d'examen, sous la vue en plongée de la caméra qui suggère la perspective divine (voir figure 9). 


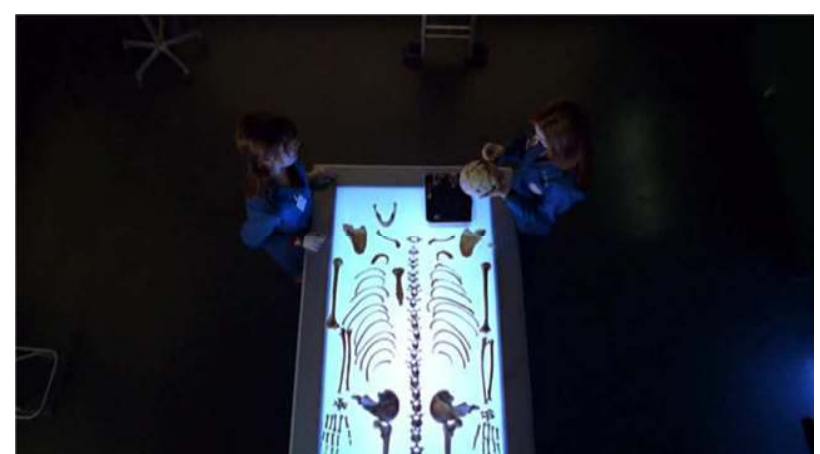

Fig. 9 : Bones, 1.8, « The Girl in the Fridge », novembre 2005

Une salle obscure percée d'un rayon de lumière bleuté, des personnages en chasuble bleue et blanche, des médecins légistes aux visages de patriarches barbus ou de madones impassibles, et au milieu un corps, des membres, à la pâleur opalescente, corps martyrisé par le scalpel dont les longues incisions accrochent le regard, ou par la violence mise en scène ${ }^{26}$. Sous son rayon bleuté, le corps iconique, emblème de l'individualisme curieusement normatif de la société de consommation, renoue à la fois avec sa matérialité biologique et avec le sentiment de sa sacralité - celle d'un individu singulier supposément échappé du jeu des compositions identitaires - personnage muet, immobile, sans rien à jouer, figure de pure représentation spéculative que reconstruit le discours de l'investigateur. Paradoxalement, le corps objet - le cadavre - suggère finalement l'être, l'individualité évanescente, corps objet libéré du jeu des incarnations ou des investissements iconiques. Regarder la réalité du cadavre alors même un cadavre représenté, un faux cadavre, évidemment - regarder le corps matériel et biologique, présenté aux regards, et rejouer constamment la scène de son auscultation minutieuse, ritualisée, c'est renouer avec le mystère de l'être, avec son potentiel de transcendance, moins comme une expérience (le mort n'est plus là, n'éprouve plus), que comme une projection, peut être fantasmée.

Cette réflexion s'est ouverte sur la question de l'émergence d'une nouvelle pornographie morbide et cathartique, elle se termine sur l'image de l'examen ritualisé du cadavre. Moins qu'une évolution, ce sont sans doute les deux faces d'une même fascination qui se dévoilent

${ }^{26}$ On notera cependant qu'on n'assiste rarement à l'autopsie pour se contenter des premières constatations, puis de la re-présentation du corps recousu, dont les organes font rarement l'objet d'une attention particulière. 
ici, alors que les cadavres n'ont pas dit leur dernier mot à l'écran (Les Revenants, en France, In the Flesh, en Grande Bretagne disent assez la fascination contemporaine pour un imaginaire morbide, selon des modalités très différentes). Entre terreur de la disparition et de la dégradation des corps, et terreur d'une existence autrefois aliénante, à présent standardisée ou virtualisée, le cadavre, dans le registre du biologique, participe d'une interrogation sur la nature - physique, biochimique, psychologique, comportementale, morale - de notre humanité et sur les modèles et discours dont elle est tributaire. Quand le personnage s'interroge sur son identité, sur sa vérité intime, dans ces nouveaux «miroirs de la vie» que sont les séries, c'est moins précisément son image qu'il regarde en miroir que son devenir cadavre, pivot d'une interrogation sur le mystère de son individualité propre, de cette âme incertaine dont il se sent dépouillé. Là où le transi invitait à se détacher de la matérialité du corps et de ses plaisirs pour mieux en libérer l'esprit, objet de toutes les attentions, les nouveaux transis fictionnels opèrent au contraire un mouvement inverse de réinvestissement spirituel de la matière, une réappropriation du corpsobjet devenu étendard d'une identité performative et standardisée. L'épisode "Butterflied », dans CSI, met ainsi en présence l'enquêtrice scientifique Sara Sidle et le cadavre d'une infirmière morte qui lui ressemble presque trait pour trait (CSI, 4.12, février 2005), son double mort. Dans la longue confrontation silencieuse qui oppose la vivante et la morte étendue sur la table d'autopsie, la très consciencieuse Sara Sidle voit l'image de son existence sacrifiée sur l'autel de son professionnalisme, et se découvre morte vivante face à un cadavre que l'enquête ramène à une vie intime riche et complexe. Bill Grissom, qui observe la scène en arrière-plan, mesure devant le même cadavre où gît celle qui pourrait être sa collègue morte, le vide sentimental de son existence. Le jeu des reflets sur les vitres déploie l'image d'une identité décomposée, à laquelle le cadavre oppose sa singularité retrouvée et le mystère de son intimité à reconstituer. Double étrange et dérangeant, le cadavre renvoie au personnage le reflet de sa réalité de corps. Ce faisant néanmoins, c'est le déficit d'âme, d'intimité propre, de l'individualité subtile qu'il a pu incarner, qui happe le personnage fasciné par cet inquiétant miroir. La métaphore filée du papillon mise en avant dans le titre de l'épisode, invoque une lecture symbolique limpide et paradoxale qui ranime l'imaginaire de l'âme dans le cadavre et lie la victime à sa résurrection fragile chez les techniciens du crime ${ }^{27}$. Mais le memento mori contemporain appelle moins à préparer son salut qu'à s'abstraire d'un univers de fonctionnalités et de consommation désincarnées, dans la fiction qui pourtant y participe,

${ }^{27}$ Un rapprochement similaire était à l'œuvre dans The Silence of the Lambs, de Jonathan Demme (1991), rapprochement que l'affiche mettait d'emblée en lumière. 
mais où l'image du cadavre, sa matérialité organique, enfonce un coin inattendu dans les représentations.

\section{L'auteure}

Emmanuelle DELANÖ̈-BRUN est maître de conférences à l'université Paris Diderot, où elle enseigne la littérature américaine, la traduction littéraire et les liens entre littérature et cinéma. Ses travaux portent sur le cinéma américain, l'adaptation littéraire, la représentation du cinéma à l'écrit et à l'écran, et les rapports entre culture populaire et culture savante que ces objets induisent. Elle a publié sur l'œuvre de Robert Altman, de Stanley Kubrick, de John Sayles, de Dashiell Hammett, de Walker Percy. Elle a co-rédigé en 2013 une étude sur The House of Mirth (roman d'Edith Wharton, adaptation de Terence Davies) avec Anne Ullmo. 